\title{
Differential Involvement of Excitatory and Inhibitory Neurons of Cat Motor Cortex in Coincident Spike Activity Related to Behavioral Context
}

\author{
David Putrino, ${ }^{1,2}$ Emery N. Brown, ${ }^{1,2}$ Frank L. Mastaglia, ${ }^{3}$ and Soumya Ghosh ${ }^{3}$ \\ ${ }^{1}$ Department of Anesthesia and Critical Care, Massachusetts General Hospital, Boston, Massachusetts 02114, ${ }^{2}$ Department of Brain and Cognitive Sciences, \\ Health Sciences and Technology Division, Harvard-Massachusetts Institute of Technology, Cambridge, Massachusetts 02139, and ${ }^{3}$ Centre for \\ Neuromuscular and Neurological Disorders, University of Western Australia, QEII Medical Centre, Nedlands, Western Australia 6009, Australia
}

To assess temporal associations in spike activity between pairs of neurons in the primary motor cortex (MI) related to different behaviors, we compared the incidence of coincident spiking activity of task-related (TR) and non-task-related (NTR) neurons during a skilled motor task and sitting quietly in adult cats (Felis domestica). Chronically implanted microwires were used to record spike activity of MI neurons in four animals (two male and two female) trained to perform a skilled reaching task or sit quietly. Neurons were identified as TR if spike activity was modulated during the task (and NTR if not). Based on spike characteristics, they were also classified as either regular-spiking (RS, putatively excitatory) or fast-spiking (FS, putatively inhibitory) neurons. Temporal associations in the activities of simultaneously recorded neurons were evaluated using shuffle-corrected cross-correlograms. Pairs of NTR and TR neurons showed associations in their firing patterns over wide areas of MI (representing forelimb and hindlimb movements) during quiet sitting, more commonly involving RS neurons. During skilled task performance, however, significantly coincident firing was seen almost exclusively between TR neurons in a smaller part of MI (representing forelimb movements), involving mainly FS neurons. The findings of this study show evidence for widespread interactions in MI when the animal sits quietly, which changes to a more specific and restricted pattern of interactions during task performance. Different populations of excitatory and inhibitory neurons appear to be synchronized during skilled movement and quiet sitting.

\section{Introduction}

Temporal associations in spike activity in the nervous system have been proposed as a mechanism for integration of distributed processes in the brain (Fetz et al., 1991; Gray, 1994; Konig and Engel, 1995; Erickson, 2001). Coincident firing activity in the motor cortex could be a marker of neural assembly formation related to representation and association of movement features. Many studies have investigated associations in spiking activity in the motor cortex (MI) and related it to shared involvement in a motor task, movement parameters, motor preparation, motor learning, and behavioral context of movement (Allum et al., 1982; Murphy et al., 1985a,b; Kwan et al., 1987; Smith and Fetz, 1989; Vaadia et al., 1995; Murthy and Fetz, 1996; Riehle et al., 1997, 2000; Donoghue et al., 1998; Lee et al., 1998; Baker et al., 2001; Grammont and Riehle, 2003; Jackson et al., 2003; Ghosh et al., 2009; Kilavik et al., 2009). However, coincident activity that is unrelated to movement tasks has also been observed in motor cortex (Murthy and Fetz, 1996; Riehle et al., 1997; Donoghue et

Received Feb. 11, 2010; revised April 11, 2010; accepted May 6, 2010.

D.P. received salary support from the National Institutes of Health under the Grants DP1-0D003646 (E.N.B.) and R01-DA015644 (E.N.B.) while writing this manuscript. We thank the Australian Neuromuscular Research Institute for support and the staff of Animal House in A Block, QEll Medical Centre, for help with animals.

Correspondence should be addressed to Dr. Soumya Ghosh, Centre for Neuromuscular and Neurological Disorders, QEll Medical Centre, A Block, 4th Floor, Nedlands, WA 6009, Australia. E-mail: sghosh@cyllene.uwa.edu.au. DOI:10.1523/JNEUROSCI.0770-10.2010

Copyright $\odot 2010$ the authors $\quad 0270-6474 / 10 / 308048-09 \$ 15.00 / 0$ al., 1998; Ghosh et al., 2009). Other studies have shown that interactions between neurons in the motor cortex are dynamic in nature, occurring during specific stages (stimulus anticipation, preparation, execution, etc.) of a motor task (Vaadia et al., 1995; Riehle et al., 1997; Baker et al., 2001).

Positron emission tomography (PET) and functional magnetic resonance imaging studies have found evidence for the formation of complex functional networks between different cortical regions during supposedly "resting states" in both motor and nonmotor areas (Biswal et al., 1995; Xiong et al., 1999; Raichle et al., 2001; Fox and Raichle, 2007; Raichle and Snyder, 2007). The findings of these studies indicate that widespread functional interactions may occur during periods of apparent inactivity - periods that have not been investigated for coincident spike activity in MI.

The motor cortex, like other neocortical areas, has a variety of excitatory and inhibitory neurons. Recent studies have distinguished spiny (excitatory) and nonspiny (inhibitory) neurons based on characteristics of extracellular spikes (McCormick et al., 1985; Kawaguchi, 1995; Cauli et al., 1997; Rao et al., 1999; Compte et al., 2000; Constantinidis and Goldman-Rakic, 2002; Swadlow, 2003; Isomura et al., 2009). Studies investigating functional interactions between fast-spiking (FS, interneuron) and regular-spiking (RS, pyramidal) neurons in motor and nonmotor areas of cerebral cortex have shown that the incidence of these interactions can vary between different neuronal subtypes (Rao 
A
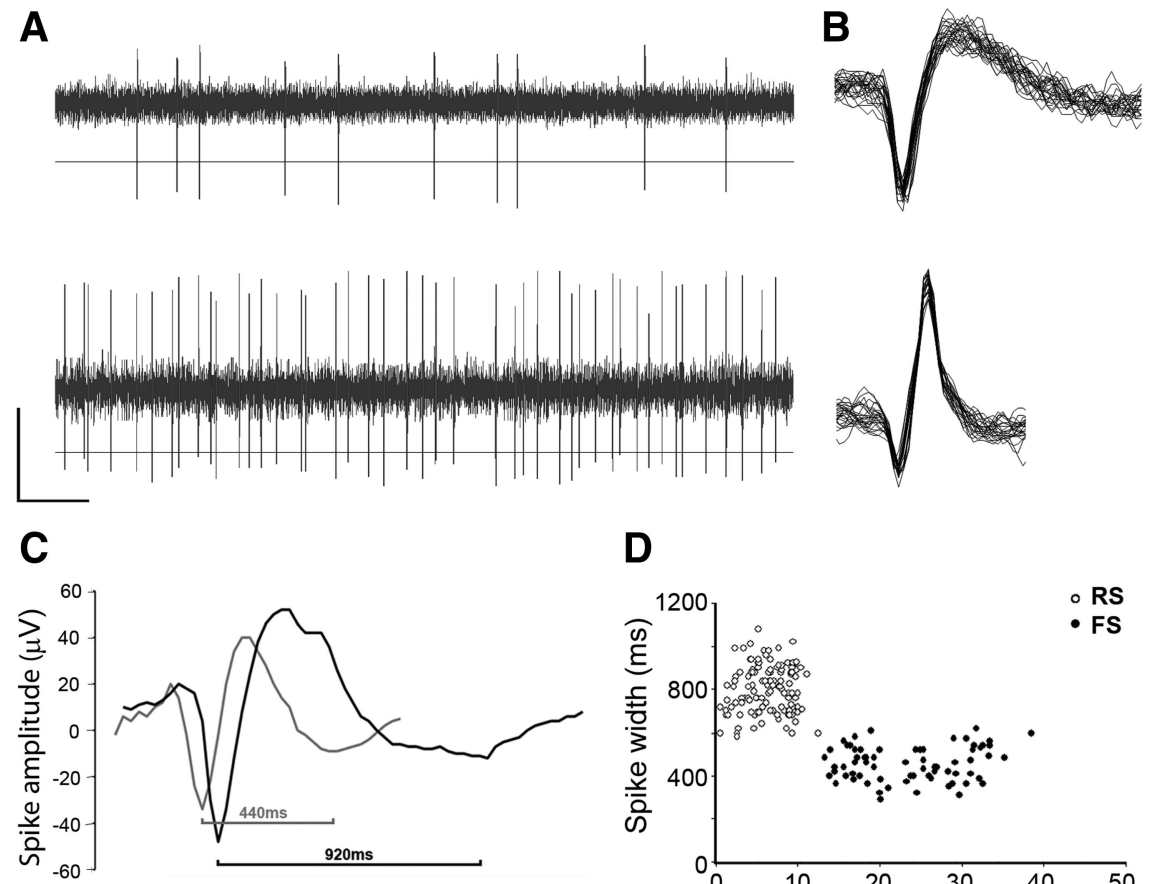

D

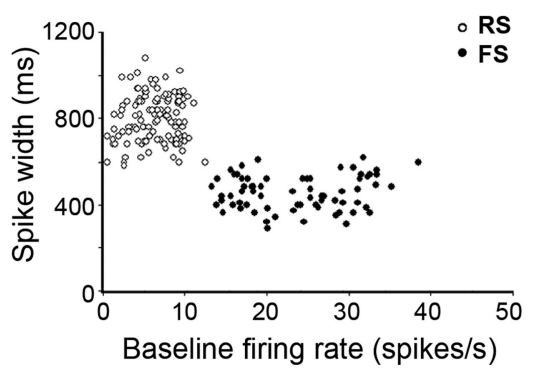

Figure 1. Spike detection and classification of RS and FS neurons. Unit isolation was performed using level detection and window discrimination. $A$, Spike trains of an RS (top trace) and FS (bottom trace) shown with a level detector. Calibration: $A, 100$ $\mu \mathrm{V}, 200 \mathrm{~ms} ; \boldsymbol{B}$ (in $\boldsymbol{A}), 150 \mu \mathrm{V}, 250 \mu$ s. $\boldsymbol{B}, 0$ verlaid waveforms of the two neurons shown alongside. $\boldsymbol{C}$, Differences in spike duration between RS (black) and FS (gray) neurons. The average spike width of each neuron was calculated after measuring the duration between the two troughs of $40-60$ spikes. $\boldsymbol{D}$, When the spike width and baseline firing rate information for all recorded neurons was plotted and compared, two clearly distinguishable neuronal populations, classified RS (open circles) and FS (filled circles) neurons, were identified.

et al., 1999; Tanaka, 1999; Compte et al., 2000; Constantinidis and Goldman-Rakic, 2002; Swadlow, 2003; Merchant et al., 2008; Isomura et al., 2009). Again, similar studies are yet to be performed during different behaviors.

In this study, therefore, we investigated coincident spike activity in MI during two different behaviors (skilled motor task and quiet sitting). We show that different populations of neurons show coincident spike activity in the different behavioral conditions. These populations vary in their function (putatively excitatory or inhibitory), topographical location in MI (forelimb or hindlimb representation), and task-related (TR) activity.

\section{Materials and Methods}

The experiments were approved by the Animal Ethics Committee of the University of Western Australia, and the National Health and Medical Research Council of Australia guidelines for the use of animal in experiments were followed throughout.

Task training and chronic microwire implantation. Methods of task training and performance, implantation of microwires in cortex, and recording spike activity have been detailed before (Ghosh et al., 2009). Briefly, four adult cats of either sex were trained to perform reaching and withdrawal movements with their forelimbs to retrieve food pellets. The animals were also trained to sit quietly after task trials were completed for further recording of spike activity from the same neurons (when no food was provided or expected). Under general anesthesia induced by intramuscular injections of ketamine $(6.6 \mathrm{mg} / \mathrm{kg}, \mathrm{i} . \mathrm{m}$.$) , xylazine (0.66 \mathrm{mg} / \mathrm{kg}$, i.m.), and pentobarbitone ( $15 \mathrm{mg} / \mathrm{kg}$, i.m.) and maintained with half the above doses of ketamine, xylazine, and pentobarbitone per hour, polytetrafluoroethylene-coated platinum-iridium microwires $(0.025 \mathrm{~mm}$ in diameter, California Wire Company, impedance $0.5-1 \mathrm{M} \Omega$ at $1000 \mathrm{~Hz}$ ) were implanted into the cortex to a depth of $\sim 1.5 \mathrm{~mm}$ into forelimb or hindlimb representations of MI [identified by intracortical microstimulation (ICMS)]. Thirtytwo microwires were implanted in each cat (16 microwires in each hemisphere, 8 in each anterior and posterior sigmoid gyri).

Video recording of task performance. A digital video camera (Cannon XL-1) was used to record task performance (at 24 frames/s) simultaneously with neural activity during all recording sessions. Video records were used to identify neural activity related to task performance and during periods when the animal was sitting quietly. Based on the video records (frame-by-frame analysis), each trial was divided into five stages: background, premovement, reach, withdraw, and feeding (Ghosh et al., 2009; see below).

Recording and analysis of neural data. A 32channel amplifier (X100, PGA32 amplifier, Multichannel Systems) and several eightchannel preamplifiers (MPA8-1, Multichannel Systems) were used to record neural activity from up to 24 microwires simultaneously on a computer using MC card and MC rack (Multichannel Systems). Trigger signals were also recorded on one analog channel of MC card from a laser beam detector, which gave brief positive and negative pulses when the beam was cut (during reach) and re-formed (during the withdrawal stage of the task), respectively. The analog trigger signal was used to identify periods of interest within large files of continuously recorded neural data. Activity in each channel was digitized at $25 \mathrm{kHz}$ (sample interval $40 \mu \mathrm{s}$ ). The same software was used to identify spike activity in each channel using threshold detectors, window discriminators, and spike shape analysis (Fig. 1). The time stamps of spike activity in each channel were exported to a Microsoft Excel spreadsheet, along with the start times of each stage of every trial. Spike activity was analyzed over a $3 \mathrm{~s}$ segment of each trial and separated into trials involving use of the left or right forelimb, or quiet sitting (no task). The time stamps of spike activity during the trials and of quiet periods were exported to NeuroExplorer software (Nex Technologies) to analyze perievent activity [perievent rasters and peristimulus time histograms (PSTHs)], mean spike frequency during different stages of the task, and temporal correlation between spike activities of neurons [crosscorrelograms (CCs)]. Statistical analysis was used to determine whether neural firing rate was significantly modulated during task performance compared to background periods (ANOVA) and to evaluate the specific task stages during which this modulation occurred (paired $t$ test). Recorded neurons were thus classified as TR if their activity modulated significantly during one or more task stages, or non-task related (NTR) if their firing rate did not alter significantly during task performance. TR neurons were further characterized as narrowly tuned (NT) or broadly tuned (BT) neurons depending on whether spike frequency was modulated (increased or decreased) during one stage or two or more stages of the task, respectively (Ghosh et al., 2009).

Classification of neurons. Neurons in the present study were classified as RS, FS, or "unspecified" based on analysis of their extracellular spiking features, but only RS and FS neurons were included in this analysis. Combined morphological and physiological studies have revealed that in most instances RS and FS neurons may be reliably identified as pyramidal neurons and inhibitory interneurons, respectively (McCormick et al., 1985; Rao et al., 1999; Tanaka, 1999; Compte et al., 2000; Constantinidis and Goldman-Rakic, 2002; Swadlow, 2003; Isomura et al., 2009). "Baseline firing rate" was defined as the average firing rate recorded from a neuron when the animal was sitting quietly. In the past, studies have reported varying baseline firing rates and spike durations for RS and FS 
neurons (McCormick et al., 1985; Swadlow, 2003). Analysis of spike duration was performed using the "Spike2" software package (Cambridge Electronic Design), and spike duration was determined using the distance between the two troughs of the action potential (Fig. 1C) so as to avoid inaccuracies that may arise from difficulties in determining the point of initial deviation from the baseline that has been previously reported (Constantinidis and Goldman-Rakic, 2002). Bursting behavior has been shown to occur in a subset of cortical neurons, and these neurons rarely show extracellular firing patterns that are particularly distinctive of either RS or FS subtypes (Calvin and Sypert, 1976; Baranyi et al., 1993; Bair et al., 1994). However, these neurons have been shown to be identifiable by computing and plotting the logarithm of each neuron's interspike interval (ISI) distribution (Nowak et al., 2003). Nonbursting and bursting neurons displayed unimodal and bimodal $\log$ (ISI) distributions, respectively. A small population including bursting neurons and others that were difficult to identify were classified as "unspecified," and excluded from the study. A recent study in the primate has discussed a class of pyramidal neuron that has a high baseline frequency but still displays the spike duration of a typical RS neuron (Merchant et al., 2008). These may have been included in some of the "unspecified" neurons in our population. When measures of spike duration and baseline firing rate of the remaining neurons were plotted together, two different populations were evident (Fig. 1). Based on this separation, we used spike duration $\geq 600 \mu$ s and baseline firing rate $<12 / \mathrm{s}$ to distinguish FS from RS neurons. The mean spike width was $803 \mu$ s (range $580-1080 \mu$ s) for RS neurons and $455 \mu$ s (range 290-620 $\mu$ s) for FS neurons, while mean spike frequency during inactivity (quiet sitting) was 6.32 spikes/s (range 0.51-12.5) for RS and 26.1 spikes/s (range 16.4-38.5) for FS neurons.

Coincident spike activity. Coincident spike activity was assessed over a $\pm 100 \mathrm{~ms}$ delay range, with bin-width resolution of $1.0 \mathrm{~ms}$. Shift predictors were computed and subsequently subtracted from raw CCs to remove artifactual correlations as a result of common task-related firing modulation. The shift predictor was averaged over all possible permutations. Such cross-correlograms have been called difference correlograms or shuffle-corrected cross-correlograms (Melssen and Epping, 1987; Gochin et al., 1991). The confidence limits were calculated independently for each bin and set at $99.9 \%$. If a bin was found to be more than double the height of the overall bin to bin variability, and at least two consecutive bins exceeded confidence limits, the peaks were considered significant (Gochin et al., 1991). No smoothing functions were applied to the CCs. Trial shuffling assumes stationarity across trials (Grün et al., 2003). We used autocorrelograms to distinguish between spike timing and latency or excitability covariations (Brody, 1999). The use of high confidence limits $(99.9 \%)$ and requirement of its crossing in at least two adjacent bins for significance was used to minimize any false-positive results. The widths of significant correlogram peaks (number of bins exceeding the $99.9 \%$ confidence limits) were classified as either narrow $(<8 \mathrm{~ms})$ or broad (>8 ms) (Gochin et al., 1991, Jackson et al., 2003), and latency of the peak was classified as centered on or near $(-2 \mathrm{~ms} \leq$ latency $\leq 2 \mathrm{~ms}$ ) the origin or at greater latency.

Coincident spike activity was evaluated during the background stage of the task, during task performance (premovement, reach, withdraw, and feed stages taken together), and during quiet sitting.
Coincident spike activity was defined as task related if it was observed during the background or other stages of the task.

Intracortical microstimulation and sensory stimulation. After the completion of recordings, electrical stimuli (ICMS, 11-16 pulses each $0.2 \mathrm{~ms}$ wide, frequency $400 \mathrm{~Hz}$, repeated every 2-4 s) were applied to the microwires to determine somatotopic representations of successful recording sites. Observable changes to neural activity in response to sensory stimulation of the forelimb (stroking the skin of the forelimb or bending the joints) were also recorded.

Perfusion and histology. Before the termination of the experiments, we made lesions in selected sites by passing $10 \mu \mathrm{A}$ for $10 \mathrm{~s}$ through the appropriate microwire. After several months of recording neural activity, the animals were killed with an overdose of anesthetic and perfused through the heart with PBS, and the brain was removed for serial sections. Coronal sections $100 \mu \mathrm{m}$ thick were cut on a freezing microtome and stained with Cresylate violet to identify microwire implant sites.

\section{Results}

\section{Task performance and quiet sitting}

Animals were trained to perform the task or sit quietly during recording sessions. Each animal developed a stereotyped technique during training that did not change from one day to the next, and most animals had a preferred forelimb that was used for the task. Each trial was divided into five stages (Ghosh et al., 2009). The background stage was the period before a food pellet 


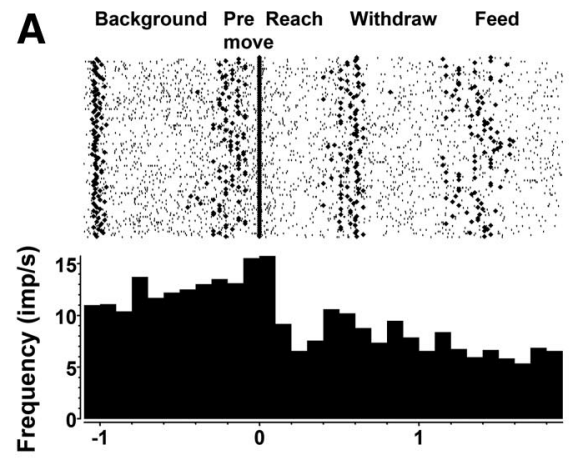

B

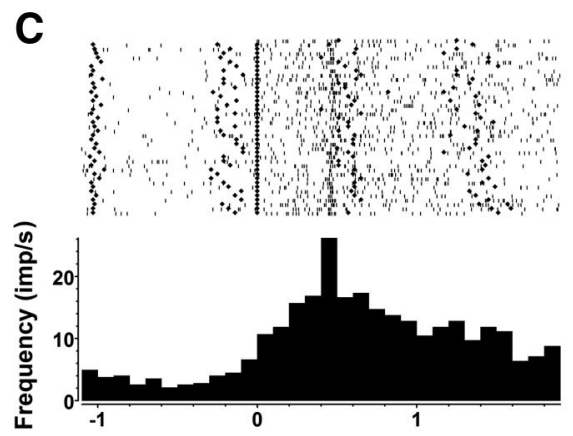

E

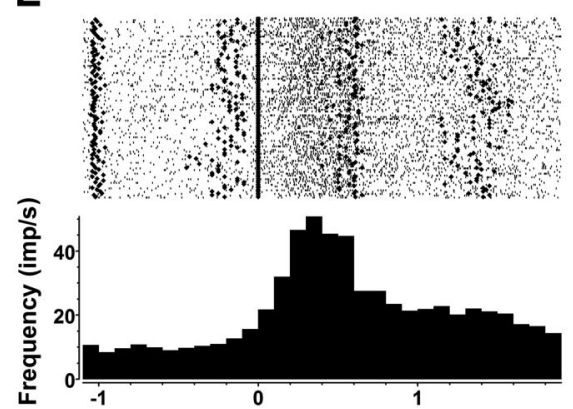

G

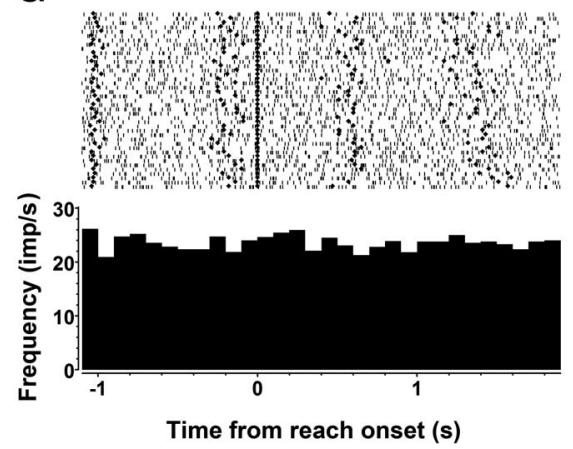

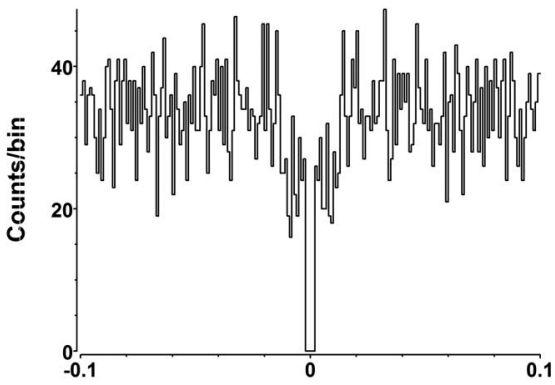

D

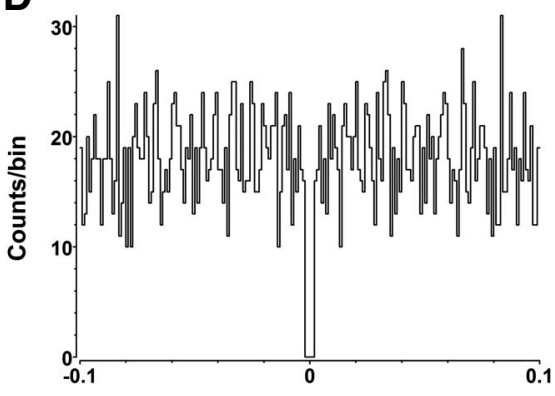

$\mathbf{F}$

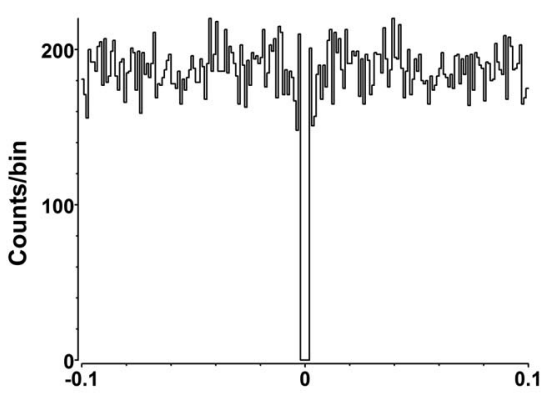

H

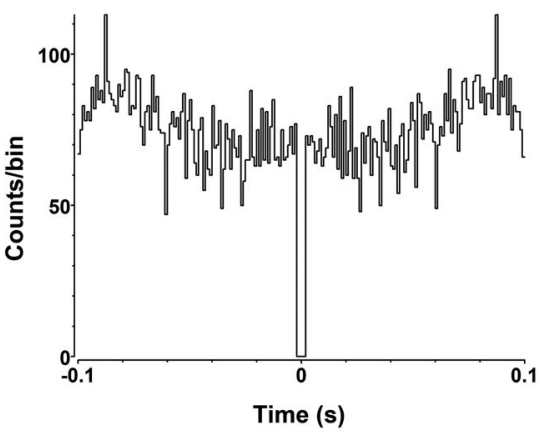

Figure 3. Examples of PSTHs and autocorrelograms of recorded neurons. Rasters and PSTHs (bin width $0.1 \mathrm{~s}$ ) of two simultaneously recorded TR neurons $(\boldsymbol{A}, \boldsymbol{C})$, as well as a simultaneously recorded pair of TR $(\boldsymbol{E})$ and NTR $(\boldsymbol{G})$ neurons, are displayed in this figure. Rasters show neural spikes (gray dots) as well as the start of each task stage (black diamonds). Rasters and PSTHs have been aligned to the start of reach. The autocorrelograms (bin width $0.001 \mathrm{~s}$ ) of the same neurons are displayed alongside $(\boldsymbol{B}, \boldsymbol{D}, \boldsymbol{F}, \boldsymbol{H})$, showing no evidence of oscillatory activity in the isolated units.

was offered in the task (and lasted $\sim 0.7-1$ s) (Fig. $2 A$ ). During this period, the animal waited expectantly for food. The preparatory (premovement or reaction time) stage occurred between the time the food was offered and the beginning of reach (averaging from 0.14 to $0.21 \mathrm{~s}$ in the 4 animals); the animal's head usually moved forward toward the food pellet as the animal leaned forward toward the task apparatus (Fig. $2 B$ ). The reach stage began when the reaching paw moved up and ended when this paw was placed over the food pellet (averaging 0.3$0.7 \mathrm{~s}$ ) (Fig. $2 \mathrm{C}$ ). The withdrawal stage began by retraction of the reaching forelimb with the food pellet under the paw and ended when the paw was lifted off the pellet in preparation to feed (averaging 0.5$0.8 \mathrm{~s}$ ) (Fig. $2 \mathrm{D}$ ). In the feeding stage (only the early part of this stage was analyzed and averaged $0.5-0.8 \mathrm{~s}$ ), the reaching forepaw was taken off the food pellet, and the head bent forward to pick up the pellet (Fig. 2E). Chewing and swallowing activity began later after the $3 \mathrm{~s}$ analysis period. After a set of task trials (50-100 trials), the animal was trained to sit quietly (Fig. $2 F$ ) for 5-10 min while we recorded spike activity related to the second behavior. During the periods of quiet sitting chosen for analysis, the animal was observed to remain alert but did not make any overt movements. During this time, no food was expected or given.

\section{Location and classification of recorded neurons}

Successful recording sites for both forelimb and hindlimb neurons were located in lamina $\mathrm{V}$ of MI (cytoarchitectonic area $4 \gamma$ ) in histological sections (Ghosh, 1997; Ghosh et al., 2009). The study compared coincident spike activity during two behavioral conditions, quiet sitting and performance of a specific task. During quiet sitting there was no modulation of the spike activity of sampled motor cortex neurons, and such activity was defined as alert, non-task-related activity. Neurons were classified into TR if spike frequency increased (Fig. 3C,E) or decreased (Fig. $3 A$ ) during one or more task stages (compared to the background stage) or NTR if spike frequency was not modulated during task performance (Fig. 3G). This does not imply that NTR neurons are not associated with motor function, since they may become active during other tasks. Autocorrelograms did not reveal any oscillatory spike activity in TR or NTR neurons (Fig. $3 B, D, F, H)$. We report on 161 TR and 63 NTR neurons recorded from forelimb (112/ 161 TR, 25/63 NTR) and hindlimb (49/161 TR and 38/63 NTR neurons) representations of the motor cortex. Neurons were classified into two subtypes based on spike duration and baseline activity rates: RS and FS neurons. In both populations of neurons, RS cells were the most commonly recorded neuronal subtype (TR: 101/161; NTR: 41/63).

\section{Associations in neural activity during quiet sitting and task performance}

Shuffle-subtracted correlograms were computed on the spike activities of simultaneously recorded NTR-NTR, NTR-TR, or TR-TR neuronal pairs during periods of task performance 
(Fig. $4 C, D, G, H$ ) or quiet sitting (Fig. $4 A, B, E, F)$. We used a mean of 60 task trials (SD 24) or $180 \mathrm{~s}$ of quiet sitting, and a mean of 2750 spikes (SD 2451) to compute each cross-correlogram. There were no differences in the numbers of task trials or spikes used to compute cross-correlograms showing significant coincident activity from those that did not. Pairs of neurons that showed coincident spike activity during task trials showed significant peaks in the cross-correlograms computed from spike activity during four stages of the task: premovement, reach, withdraw, and feed. In a small number of neural pairs, coincident activity was also seen during the background stage (Ghosh et al., 2009), and such activity was also considered task related (anticipatory activity) (see Riehle et al., 1997).

The pattern of interactions was different in the two behavioral states (Fig. 5): during quiet sitting, 84 of 1402 pairs of all neurons tested $(6 \%)$ showed significant peaks, while during task performance increased frequency of interactions was observed among TR neurons (59/550 pairs tested, 11\%) and fewer interactions seen involving NTR neurons (16/852 pairs tested, 2\%). Seven of 136 NTR-NTR pairs, 71 of 716 NTR-TR pairs, and 6 of 550 TR-TR pairs showed significant evidence of temporal associations in their spiking during quiet sitting. Significantly fewer (14/716) NTR-TR neuronal pairs $\left(p<0.001, \chi^{2}\right.$ analysis) and significantly more (59/550) TR-TR neuronal pairs showing evidence of coincident firing during task performance $\left(p<0.001, \chi^{2}\right.$ analysis) when compared to control periods. A small proportion of neuronal pairs tested showed coincident firing during both quiet sitting and task performance.

Associations between neural subtypes Neurons were classified into two subtypes based on spike duration and baseline activity rates: RS (putatively excitatory) and FS (putatively inhibitory) neurons. In both populations of neurons, RS cells were the most commonly recorded neuronal subtype (TR: 101/161; NTR: 41/63). Correlated pairs were divided into three categories based on the involvement of neuronal subtypes: RS-RS, FS-FS, or RSFS. Among the TR-TR neuronal associations during task performance, FS-FS pairs were the most common, followed by RS-FS, and RS-RS were the least common (Fig. $6 C)\left(p<0.001, \chi^{2}\right.$ analysis). The NTR-TR interactions showed a different trend during quiet sitting and task performance, with RS-FS pairs being the most

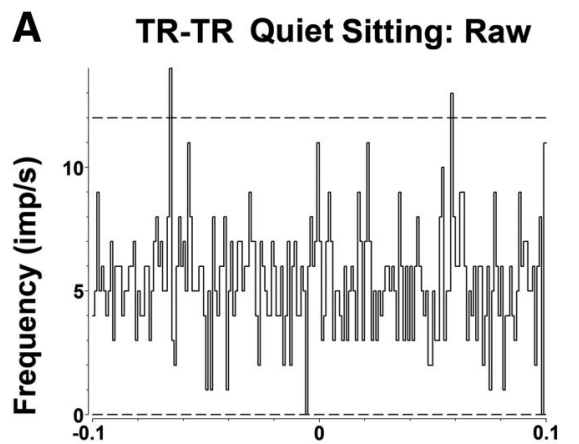

B TR-TR Quiet Sitting: Corrected
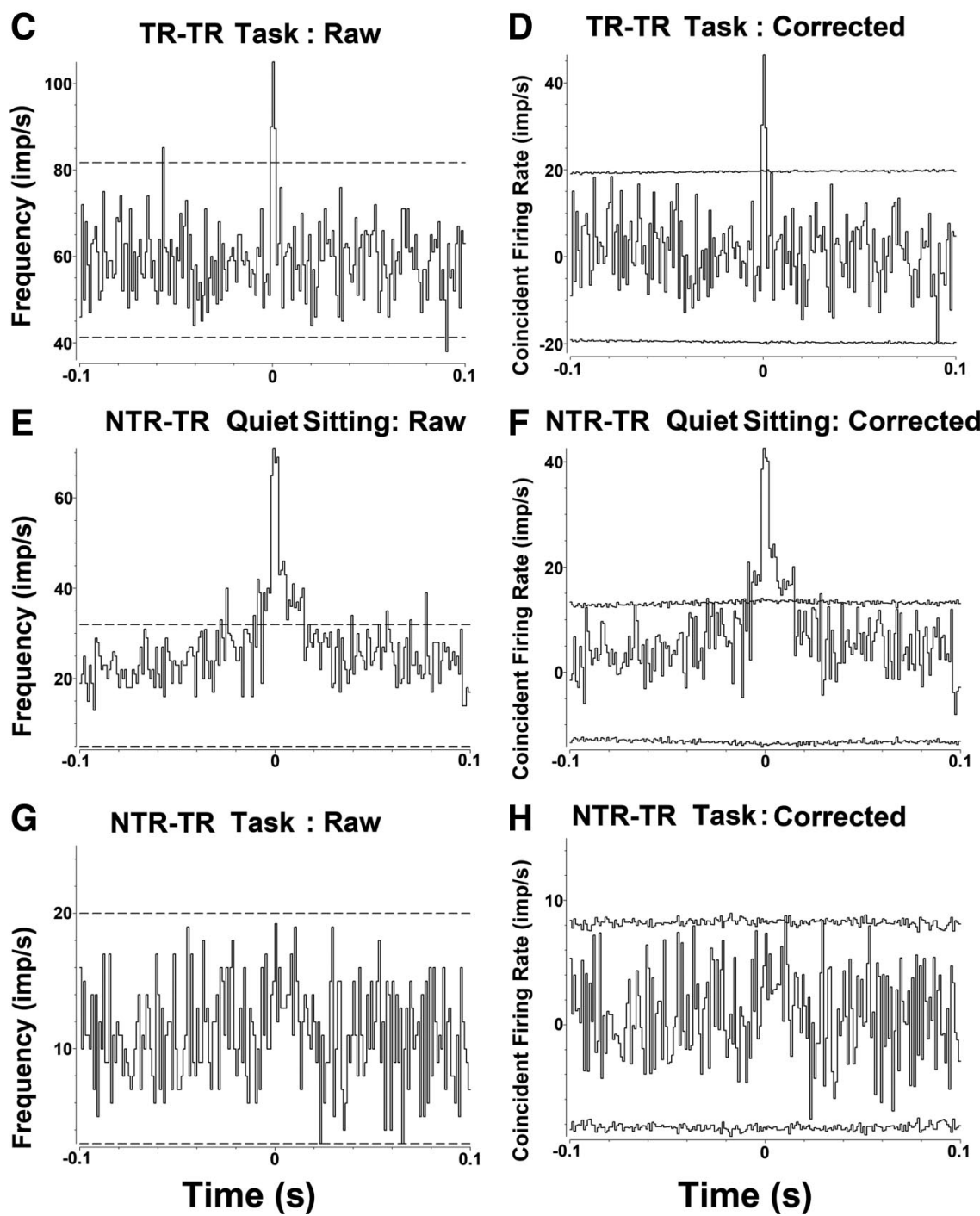

Figure 4. Examples of raw and shuffle-subtracted cross-correlograms computed between pairs of neurons during quiet sitting and task performance. Raw $(\boldsymbol{A}, \boldsymbol{C}, \boldsymbol{E}, \boldsymbol{G})$ and shuffle-corrected $(\boldsymbol{B}, \boldsymbol{D}, \boldsymbol{F}, \boldsymbol{H})$ cross-correlograms (bin width $0.001 \mathrm{~s})$ were computed between the simultaneously recorded pairs of neurons displayed in Figure 3 during quiet sitting $(A, B, E, F)$ and task performance $(\boldsymbol{C}, \boldsymbol{D}, \boldsymbol{G}, \boldsymbol{H})$. For examining coincident spike activity during task performance, cross-correlograms were computed for the following stages of the task: premovement, reach, withdraw, and feed. Correlograms computed for the background stage are not shown. Note the differences between the raw and shuffle corrected cross-correlograms after subtraction of the "shift predictor." The shift predictor was averaged over all possible permutations. Note the narrow peak that reaches significance in the second correlogram $(\boldsymbol{D})$, indicating a significant firing pattern association between TR-TR neurons occurring specifically during task performance. Next, consider the correlograms of simultaneously recorded NTR and TR neurons, during the quiet sitting $(\boldsymbol{F})$ and task $(\boldsymbol{H})$ periods. Note that in this case, there is a broad peak that reaches significance in the first correlogram $(\boldsymbol{F})$, indicating a significant firing pattern association that occurs during quiet sitting. 


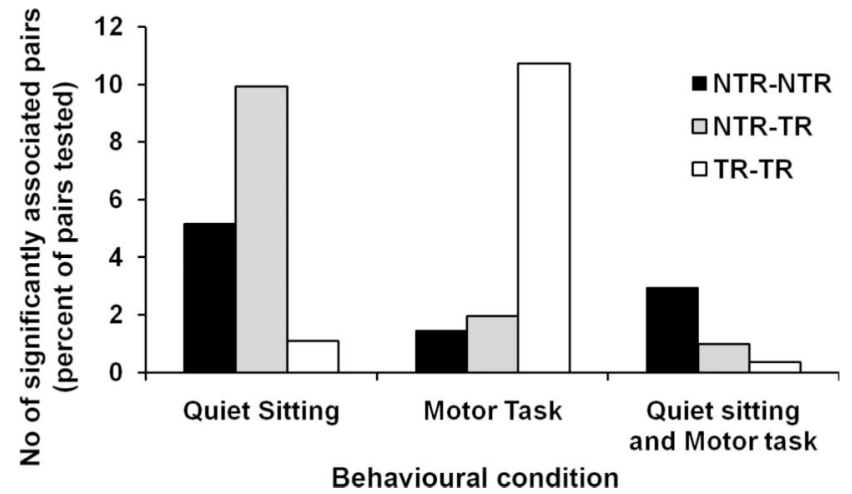

Figure 5. Incidence of significant associations in neural activity during different behavioral conditions. The number of neuronal pairs that showed significantly coincident spike activity (expressed as a percentage of the total number of tested pairs) is illustrated as histograms for NTR-NTR, NTR-TR, and TR-TR neuronal pairs during different behavioral conditions.

common, followed by RS-RS, and FS-FS pairs being the least common (Fig. $4 B)\left(p<0.005, \chi^{2}\right.$ analysis). NTR-NTR pairs showed FS-FS and FS-RS pairs in similar proportions, but fewer RS-RS interactions during both quiet sitting and task performance.

\section{Topography of neural associations}

The neurons involved in NTR-TR, NTR-NTR, and TR-TR neuronal pairings were recorded from forelimb and hindlimb representations in MI. Significantly associated neuronal pairs were separated into groups where both cells were recorded from the hindlimb representation (HL-HL), both recorded from the forelimb representation (FL-FL), or one cell from each (FL-HL). During task performance, the majority of significant associations were seen between FL-FL neuronal pairs, while during quiet sitting associations were approximately equally divided between FL-FL, FL-HL, and HL-HL pairs (Fig. 7A). Among TR-TR pairs, the overwhelming majority of interactions $(64 / 65)$ were found to be FL-FL pairings, a finding that was independent of the physical distance between recording sites (Fig. $7 B$ ). In contrast, the NTR-TR pairs showed approximately equal rates of association between FL-FL, FL-HL, and HL-HL cell pairs (Fig. 7B). Incidence of coincident firing of NTR-NTR pairs showed evidence of interactions between FL-HL and HL-HL cell pairs, but none between FL-FL pairs (Fig. 7B).

\section{Peak widths and latencies of correlograms}

The correlogram peak widths seen in NTR-NTR, NTR-TR, and TR-TR pairings with significant evidence of coincident firing patterns are shown in Figure $8 A$. Most peaks were narrow (2-8 $\mathrm{ms})$. The few correlograms involving NTR-NTR pairings did not show any significant trends in their peak widths, with equal proportions of neuronal pairs showing narrow (6/13) or broad (7/ 13) peaks. Overall, correlograms involving NTR-TR pairings showed a variety of peak widths, but narrow peaks were more frequent (57/93). However, all of the NTR-TR neuronal pairs that showed significant associations in their firing patterns exclusively during movement periods had narrowly peaked correlograms. Among TR-TR pairs, the correlograms were almost exclusively narrow peaked (55/65). The few TR-TR neuronal pairs that showed coincident firing patterns during quiet sitting, however, showed broad correlogram peaks (5/6). Thus, during task performance, coincident activity between pairs of neurons (whether NTR or TR) was almost always characterized by narrow
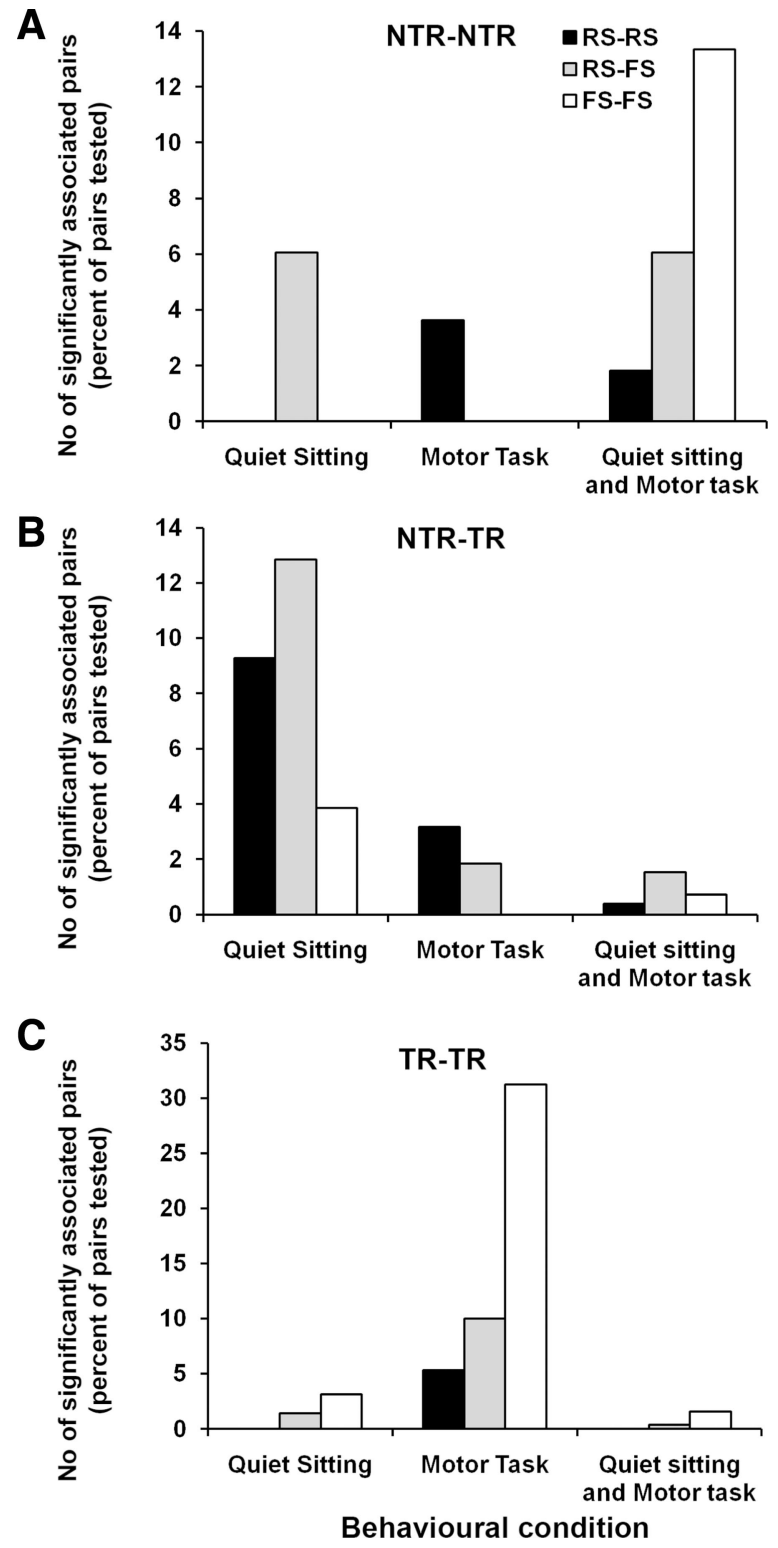

Figure 6. Effect of cell subtype and task-related function on incidence of correlation. The frequency (expressed as a percentage of the total number of tested pairs) of significant associations in neural activity observed among different combinations of neuronal subtypes (RS, FS) is illustrated in NTR-NTR $(\boldsymbol{A})$, NTR-TR $(\boldsymbol{B})$, and TR-TR $(\boldsymbol{C})$ neuronal pairings during different behavioral conditions.

correlogram peaks, whereas during quiet sitting both narrow and broad peaks were seen equally frequently. The peak latencies of all significant correlograms were mainly at or near origin (Fig. 8 B).

\section{Discussion}

The study compares coincident spike activity between pairs of MI neurons during performance of a skilled task and during periods of quiet sitting and shows that different populations of neurons are synchronized during the different behaviors (Fig. 9). During periods of quiet sitting, coincidences are observed in wider areas of MI involving forelimb and hindlimb representations. This is replaced by a more restricted pattern of coincident activity (involving task-related neurons in the forelimb area) during skilled movement. Different neuronal subtypes (putatively excitatory and inhibitory) are preferentially involved in coincident activity observed in the two behavioral conditions. 

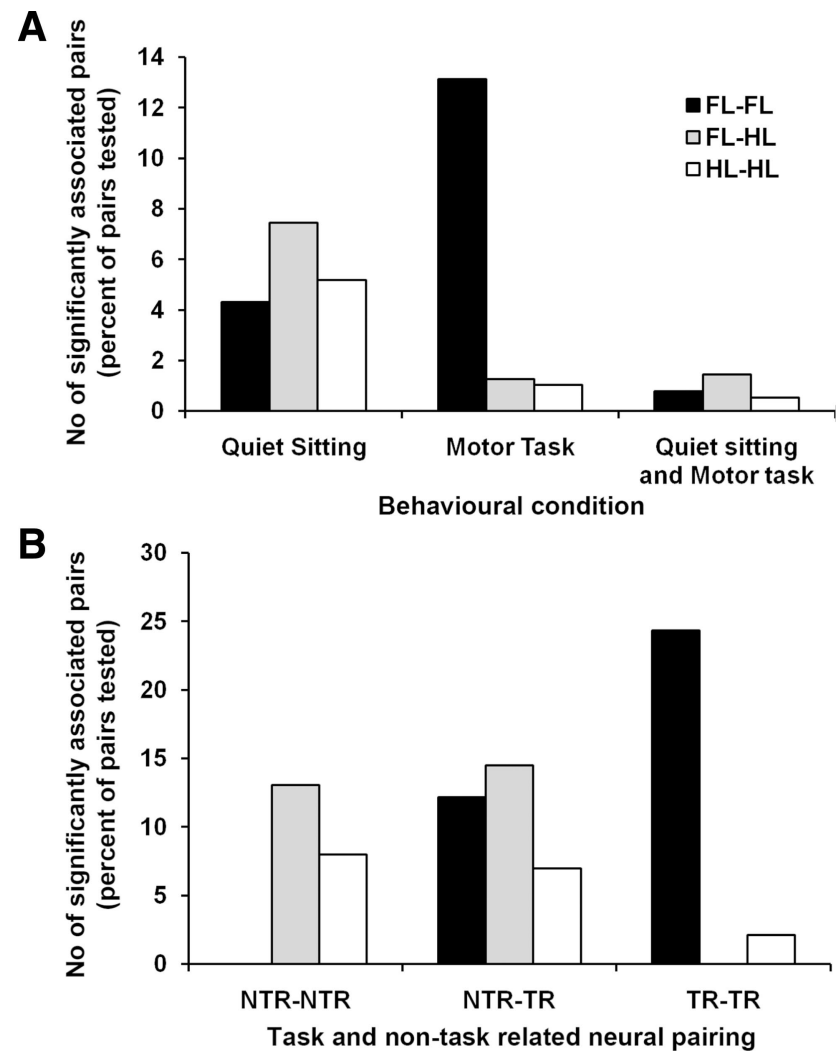

Figure 7. Limb representation and the incidence of correlation. $A$, Differences in the rates (as a percentage of the total number of tested pairs) of coincident firing patterns are seen when neuronal pairs are grouped according to limb representation as $F L-F L, ~ H L-F L$, or $H L-H L$ during different behavioral conditions. $\boldsymbol{B}$, The pairing of neurons as NTR-NTR, NTR-TR, or TR-TR appeared to influence the incidence of associations that were detected between $\mathrm{FL}-\mathrm{FL}$, $\mathrm{HL}-\mathrm{FL}$, or HL-HL pairings.

\section{Differences in interactions between RS and FS neurons}

Classification of units using extracellular spike characteristics into two categories (RS, putatively excitatory neurons, and FS, putatively inhibitory interneurons) is an oversimplification. Anatomical and physiological studies show a large variety of neuronal types in the cortex (McCormick et al., 1985; Kawaguchi, 1995; Cauli et al., 1997; Swadlow, 2003), and it is likely that a small proportion of neurons in our sample were misclassified. However, the differences in the observed subtype interactions in this study are large and unlikely to be explained by these errors. Previous studies in prefrontal (Constantinidis and Goldman-Rakic, 2002) and sensory (Swadlow, 2003) areas have found that synchronized activity between FS neurons is more common than RS neurons during task performance or sensory stimulation. This study found similar bias in interactions in MI during task performance, but not during quiet sitting.

\section{Differences in coincident spike activity during quiet sitting} and task performance

Neural synchrony in MI is seen during a variety of tasks in MI during skilled movement (Allum et al., 1982; Murphy et al., 1985a,b; Kwan et al., 1987; Smith and Fetz, 1989; Vaadia et al., 1995; Murthy and Fetz, 1996; Riehle et al., 1997; Donoghue et al., 1998; Lee et al., 1998; Baker et al., 2001; Jackson et al., 2003; Ghosh et al., 2009). Oscillations of local field potentials have been observed in MI during task performance (Baker et al., 2001; Cheyne et al., 2008) and may contribute to neural synchrony. However, other studies have related neural synchrony to nonmo-
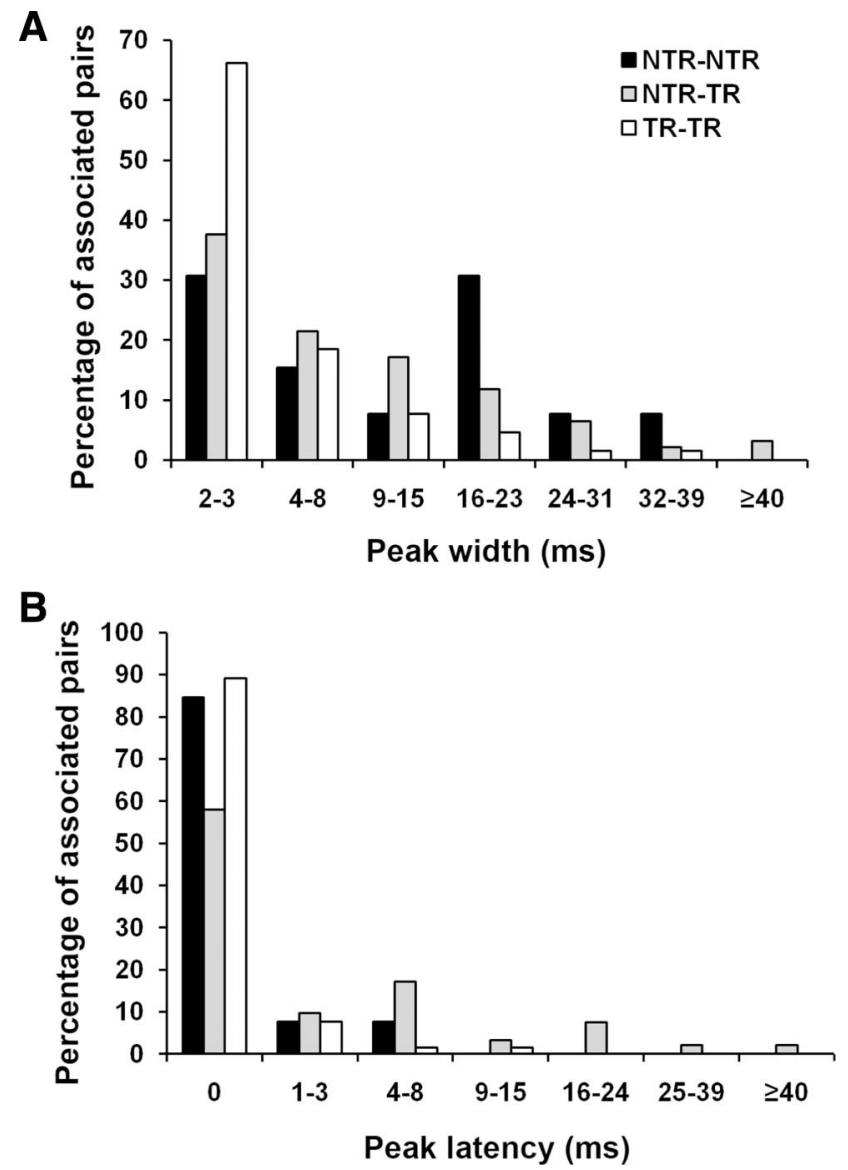

Figure 8. Distribution of peak widths $(\boldsymbol{A})$ and latencies $(\boldsymbol{B})$ for all of the correlograms that reached significance in this study. The pairings of different neuronal types (NTR-NTR, NTR-TR, and TR-TR) are indicated by the different bar colors, and the numbers of peak latencies and widths are represented as percentages of the total number of significant associations in each pairing.

tor functions such as focused attention, showing that local oscillatory field potentials and associated coincident spike activity in $\mathrm{MI}$ are prominent during alert quiet periods (when the animal is not specifically engaged in a task), but suppressed during trained movements (Murthy and Fetz, 1996; Donoghue et al., 1998). This study shows that coincident activity is present during movement and quiet periods but they involve different populations of neurons. The pattern of associations observed during quiet sitting was widespread and involved neurons with more varied function. The function of neuronal interactions in the cortex during supposedly resting states is still debated, and has been proposed to reflect balanced activity of neural circuits and predictions of environmental demands (Raichle and Snyder, 2007). The neuronal synchrony observed during the task performance may reflect a change of this balance to preferentially involve task-related FS neurons. Thus, neural interactions during different behaviors involve different populations of cortical neurons, varying in their spatial distribution, their task-related activity, and their functional role (excitatory vs inhibitory).

\section{Correlogram peaks}

During task performance, significant correlations were almost exclusively narrow peaked. Correlogram peaks that are narrow and centered on the origin are a frequent correlation pattern observed between cells in the cerebral cortex (Gochin et al., 1991; Konig and Engel, 1995; Jackson et al., 2003), and are thought to 


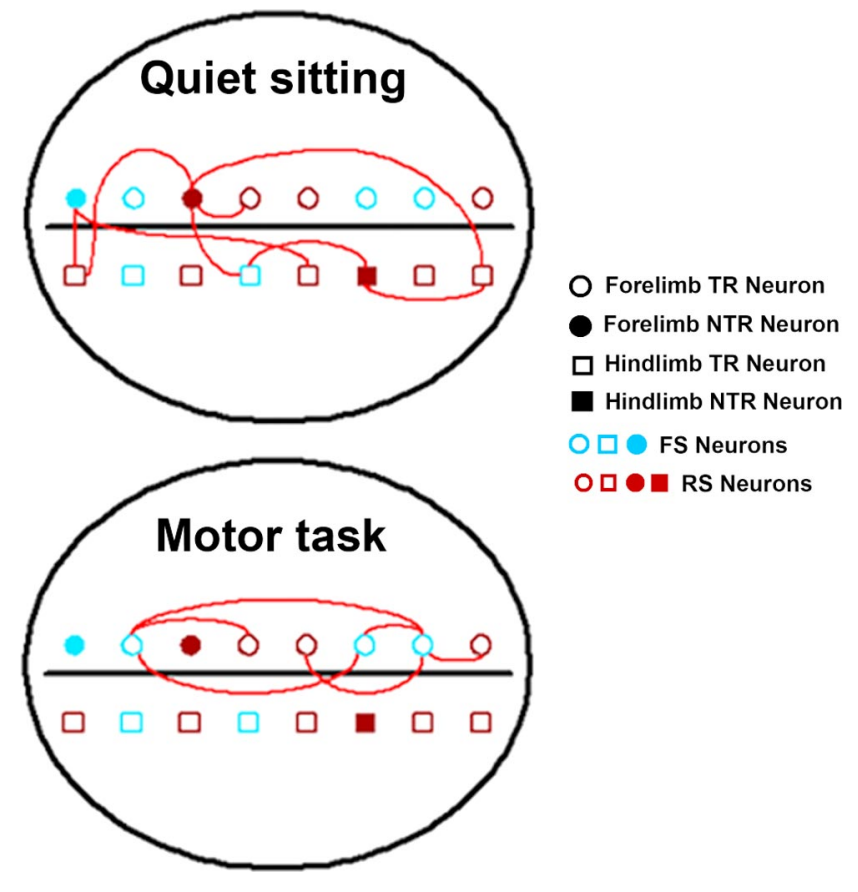

Figure 9. Significant associations in MI neural networks. Pictorial representation of the pattern of coincident spike activities seen between neurons with different functions during a typical recording session from one hemisphere. The horizontal black line is a simplified representation of the cruciate sulcus, which divides the cat MI anatomically into rostral and caudal $4 \gamma$. Each recorded neuron has three attributes indicated by the shape (circle, forelimb representation; square, hindlimb representation), filling (filled, NTR; unfilled, TR), and color (blue, FS; red, RS) of the symbol. In this recording, during quiet sitting we see more varied interactions between NTR and TR neuronal pairs, between FL and HL neurons, and between $\mathrm{RS}-\mathrm{RS}$ and RS-FS pairs. In contrast, during task performance we see that interactions are limited to those between TR neurons from forelimb representations, and FS-FS or RS-FS pairs.

indicate either common excitatory input or reciprocal excitatory connections between the two regions (Moore et al., 1970; Melssen and Epping, 1987; Fetz et al., 1991; Gochin et al., 1991; Konig and Engel, 1995). Conversely, correlogram peaks due to inhibitory input are typically broad, and less commonly seen (Moore et al., 1970; Melssen and Epping, 1987; Gochin et al., 1991; Konig and Engel, 1995). Interestingly, neuronal pairs that were correlated during inactivity showed a greater frequency of broad peaks than those that were correlated during task performance.

\section{Spatial distribution of coincident neural activity}

Many studies in MI have found that neuronal pairs showing coincident spike activity during task performance are located within a few millimeters of each other in a small region of MI representing a single limb or body part (Allum et al., 1982; Murphy et al., 1985a,b; Kwan et al., 1987; Smith and Fetz, 1989; Vaadia et al., 1995; Ghosh et al., 2009). Coincident activity between neurons in different limb areas was not seen when specifically examined (Ghosh et al., 2009). On the other hand, coincident activity studied during periods of quiet sitting involved larger interelectrode distances and more extensive regions of MI (wherever electrodes were implanted) and included interactions between neurons in different limb representations. These interactions were not associated with modulation of neuronal activity. Other studies have shown that during cognitive motor processing, neural interactions are not accompanied by modulation of spike frequency (Vaadia et al., 1995; Riehle et al., 1997).

\section{Function of synchronized spike activity}

Synchronized spike activity is thought to reflect network interactions between neurons through direct synaptic interactions or common synaptic inputs. In MI these interactions have been found to link together neurons with common involvement in a task (Allum et al., 1982; Murphy et al., 1985a,b; Kwan et al., 1987; Smith and Fetz, 1989; Vaadia et al., 1995; Baker et al., 2001; Jackson et al., 2003; Ghosh et al., 2009). Interactions between excitatory and inhibitory interneurons are thought to play a role in tuning of neurons to sensorimotor parameters, surround inhibition, and organization of cortical columns (Rao et al., 1999; Swadlow, 2003). Less is known about neuronal interactions unrelated to specific task performance, although MRI and PET studies have shown increased activity and interactions of brain areas during quiet resting, which is diminished during specific goal-related behaviors (Biswal et al., 1995; Xiong et al., 1999; Raichle et al., 2001; Fox and Raichle, 2007; Raichle and Snyder, 2007). This is the first study to examine coincident spike activity and neural interactions in MI during the "resting state" and compare that with the interactions that occur during motor tasks. Clearly different patterns of interactions were observed during the different behavioral states, suggesting that coincident spike activity is affected by multiple functions in MI and other cortical areas.

\section{References}

Allum JH, Hepp-Reymond M-C, Gysin R (1982) Cross-correlation analysis of interneuronal connectivity in the motor cortex of the monkey. Brain Res 231:325-334.

Bair W, Koch C, Newsome W, Britten K (1994) Power spectrum analysis of bursting cells in area MT in the behaving monkey. J Neurosci 14:2870-2892.

Baker SN, Spinks R, Jackson A, Lemon RN (2001) Synchronization in monkey motor cortex during a precision grip task. I. Task dependant modulation in single unit synchrony. J Neurophysiol 85:869-885.

Baranyi A, Szente MB, Woody CD (1993) Electrophysiological characterization of different types of neurons recorded in vivo in the motor cortex of the cat. I. Patterns of firing activity and synaptic responses. J Neurophysiol 69:1850-1864.

Biswal B, Yetkin FZ, Haughton VM, Hyde JS (1995) Functional connectivity in the motor cortex of resting human brain using echo-planar MRI. Magn Reson Med 34:537-541.

Brody CD (1999) Correlations without synchrony. Neural Comput 11 1537-1551.

Calvin WH, Sypert GW (1976) Fast and slow pyramidal tract neurons: an intracellular analysis of their contrasting repetitive firing properties in the cat. J Neurophysiol 39:420-434.

Cauli B, Audinat E, Lambolez B, Angulo MC, Ropert N, Tsuzuki K, Hestrin S, Rossier J (1997) Molecular and physiological diversity of cortical nonpyramidal cells. J Neurosci 17:3894-3906.

Cheyne D, Bells S, Ferrari P, Gaetz W, Bostan AC (2008) Self paced movements induce high-frequency gamma oscillations in primary motor cortex. Neuroimage 42:332-342.

Compte A, Brunel N, Goldman-Rakic PS, Wang XJ (2000) Synaptic mechanisms and network dynamics underlying spatial working memory in a cortical network model. Cereb Cortex 10:910-923.

Constantinidis C, Goldman-Rakic PS (2002) Correlated discharges among putative pyramidal neurons and interneurons in the primate prefrontal cortex. J Neurophysiol 88:3487-3497.

Donoghue JP, Sanes JN, Hatsopoulos NG, Gaál G (1998) Neuronal discharge and local field potential oscillations in primate motor cortex during voluntary movements. J Neurophysiol 79:159-173.

Erickson RP (2001) The evolution and implication of population and modular neural coding ideas. Prog Brain Res 130:9-29.

Fetz E, Toyama K, Smith W (1991) Synaptic interactions between cortical neurons. In: Cerebral cortex (Peters A, Jones EG, eds), pp 1-47. New York: Plenum.

Fox MD, Raichle ME (2007) Spontaneous fluctuations in brain activity observed with functional magnetic resonance imaging. Nat Rev Neurosci $8: 700-711$

Ghosh S (1997) Identification of motor areas of the cat cerebral cortex based on studies of cortical stimulation and corticospinal connections. J Comp Neurol 380:191-214. 
Ghosh S, Putrino D, Burro B, Ring A (2009) Patterns of spatio-temporal correlations in the neural activity of the cat motor cortex during trained forelimb movements. Somatosens Mot Res 26:31-49.

Gochin PM, Miller EK, Gross CG, Gerstein GL (1991) Functional interactions among neurons in inferior temporal cortex of the awake macaque. Exp Brain Res 84:505-516.

Grammont F, Riehle A (2003) Spike synchronization and firing rate in a population of motor cortical neurons in relation to movement direction and reaction time. Biol Cybern 88:360-373.

Gray CM (1994) Synchronous oscillations in neuronal systems: mechanisms and functions. J Comput Neurosci 1:11-38.

Grün S, Riehle A, Diesmann M (2003) Effect of cross-trial nonstationarity on joint-spike events. Biol Cybern 88:335-351.

Isomura Y, Harukuni R, Takekawa T, Aizawa H, Fukai T (2009) Microcircuitry coordination of cortical motor information in self-initiation of voluntary movements. Nat Neurosci 12:1586-1593.

Jackson A, Gee VJ, Baker SN, Lemon RN (2003) Synchrony between neurons with similar muscle fields in monkey motor cortex. Neuron 38:115-125.

Kawaguchi Y (1995) Physiological subgroups of nonpyramidal cells with specific morphological characteristics in layer II/III of rat frontal cortex. J Neurosci 15:2638-2655.

Kilavik BE, Roux S, Ponce-Alvarez A, Confais J, Grün S, Riehle A (2009) Long-term modifications in motor cortical dynamics induced by intensive practice. J Neurosci 29:12653-12663.

König P, Engel AK (1995) Correlated firing in sensory-motor systems. Curr Opin Neurobiol 5:511-519.

Kwan HC, Murphy JT, Wong YC (1987) Interaction between neurons in precentral cortical zones controlling different joints. Brain Res 400: 259-269.

Lee D, Port NL, Kruse W, Georgopoulos AP (1998) Variability and correlated noise in the discharge of neurons in motor and parietal areas of the primate cortex. J Neurosci 18:1161-1170.

McCormick DA, Connors BW, Lighthall JW, Prince DA (1985) Comparative electrophysiology of pyramidal and sparsely spiny stellate neurons of the neocortex. J Neurophysiol 54:782-806.

Melssen WJ, Epping WJM (1987) Detection and estimation of neuronal connectivity based on crosscorrelation analysis. Biol Cybern 57:403-414.

Merchant H, Naselaris T, Georgopoulos AP (2008) Dynamic sculpting of directional tuning in the primate motor cortex during three-dimensional reaching. J Neurosci 28:9164-9172.
Moore GP, Segundo JP, Perkel DH, Levitan H (1970) Statistical signs of synaptic interaction in neurons. Biophys J 10:876-900.

Murphy JT, Kwan HC, Wong YC (1985a) Cross correlation studies in primate motor cortex: synaptic interaction and shared input. Can J Neurol Sci 12:11-23.

Murphy JT, Kwan HC, Wong YC (1985b) Cross correlation studies in primate motor cortex: event related correlation. Can J Neurol Sci 12:24-30.

Murthy VN, Fetz EE (1996) Synchronization of neurons during local field potential oscillations in sensorimotor cortex of awake monkeys. J Neurophysiol 76:3968-3982.

Nowak LG, Azouz R, Sanchez-Vives MV, Gray CM, McCormick DA (2003) Electrophysiological classes of cat primary visual cortical neurons in vivo as revealed by quantitative analyses. J Neurophysiol 89:1541-1566.

Raichle ME, Snyder AZ (2007) A default mode of brain function: a brief history of an evolving idea. Neuroimage 37:1083-1090.

Raichle ME, MacLeod AM, Snyder AZ, Powers WJ, Gusnard DA, Shulman GL (2001) A default mode of brain function. Proc Natl Acad Sci U S A 98:676-682.

Rao SG, Williams GV, Goldman-Rakic PS (1999) Isodirectional tuning of adjacent interneurons and pyramidal cells during working memory: evidence for microcolumnar organization in PFC. J Neurophysiol 81:19031916.

Riehle A, Grün S, Diesmann M, Aertsen A (1997) Spike synchronization and rate modulation differentially involved in motor cortical function. Science 278:1950-1953.

Riehle A, Grammont F, Diesmann M, Grün S (2000) Dynamical changes and temporal precision of synchronized spiking activity in monkey motor cortex during movement preparation. J Physiol Paris 94:569-582.

Smith WS, Fetz EE (1989) Effects of synchrony between primate corticomoto-neuronal cells on post-spike facilitation of muscles and motor units. Neurosci Lett 96:76-81.

Swadlow HA (2003) Fast-spike interneurons and feedforward inhibition in awake sensory neocortex. Cereb Cortex 13:25-32.

Tanaka S (1999) Architecture and dynamics of the primate prefrontal cortical circuit for spatial working memory. Neural Netw 12:1007-1020.

Vaadia E, Haalman I, Abeles M, Bergman H, Prut Y, Slovin H, Aertsen A (1995) Dynamics of neuronal interactions in monkey cortex in relation to behavioural events. Nature 373:515-518.

Xiong J, Parsons LM, Gao J-H, Fox PT (1999) Interregional connectivity to primary motor cortex revealed using MRI resting state images. Hum Brain Mapp 8:151-156. 